\title{
Filling factor dependence of the fractional quantum Hall effect gap
}

\author{
V. S. Khrapai, A. A. Shashkin, M. G. Trokina, and V. T. Dolgopolov \\ Institute of Solid State Physics, Chernogolovka, Moscow District 142432, Russia \\ V. Pellegrini and F. Beltram \\ NEST INFM-CNR, Scuola Normale Superiore, Piazza dei Cavalieri 7, I-56126 Pisa, Italy \\ G. Biasiol and L. Sorba* \\ Laboratorio Nazionale TASC-INFM and NEST INFM-CNR, I-34012 Trieste, Italy \\ * Scuola Normale Superiore, Piazza dei Cavalieri 7, I-56126 Pisa, Italy
}

\begin{abstract}
We directly measure the chemical potential jump in the low-temperature limit when the filling factor traverses the $\nu=1 / 3$ and $\nu=2 / 5$ fractional gaps in two-dimensional (2D) electron system in $\mathrm{GaAs} / \mathrm{AlGaAs}$ single heterojunctions. In high magnetic fields $B$, both gaps are linear functions of $B$ with slopes proportional to the inverse fraction denominator, $1 / q$. The fractional gaps close partially when the Fermi level lies outside. An empirical analysis indicates that the chemical potential jump for an ideal 2D electron system, in the highest accessible magnetic fields, is proportional to $q^{-1} B^{1 / 2}$.

PACS numbers: 73.43.Fj, 73.21.-b, 73.40.Kp
\end{abstract}

The fractional quantum Hall effect in two-dimensional (2D) electron systems [1] is believed to be a many-body phenomenon. The dissipationless state manifested by zeros in the longitudinal resistance and plateaus in the Hall resistance forms at fractional filling factors of Landau levels, $\nu=p / q$, and the gap is predicted to be caused by electron-electron interactions (see Ref. [2] and references therein). According to numerical calculations, the chemical potential discontinuity corresponding to the fractional gap is determined by $e^{2} / \varepsilon l_{B}$ (where $\varepsilon$ is the dielectric constant and $l_{B}=(\hbar c / e B)^{1 / 2}$ is the magnetic length), being almost independent of the fraction, or the denominator $q$, for $1 / 3 \leq \nu \leq 2 / 3[3,4,[4]$. For quasiparticles with fractional charge $e / q[6]$, the activation energy is $q$ times smaller than the chemical potential discontinuity. This determines the hierarchy of fractions, as inferred from the concept of composite fermions [7]. Notably, earlier studies predicted a reduction of the chemical potential discontinuity with increasing $q$ for the same sequence [8].

The fractional gap being small, its experimental determination is pretty demanding with respect to both samples and methods. Attempts to experimentally estimate the fractional gap value yielded similar results in high magnetic fields [9, 10, 11, 12, 13, 14] and, therefore, it is unlikely that the gap is strongly influenced by the residual disorder in the 2D electron system. Standard measurements of activation energy at the longitudinal resistance minima were used to probe the fractional gaps with different denominators [9, 15, 16]. However, transport studies yield a mobility gap which may be different from the gap in the spectrum. The latter can be determined using direct thermodynamic measurements of the chemical potential jump across the gap, similar to those of Refs. [11, 17]. Recent thermodynamic studies showed that in the completely spin-polarized regime, the gap value for $\nu=1 / 3$ is equal to that for $\nu=2 / 3$, reflecting the electron-hole symmetry in the spin-split Landau level, and increases linearly with magnetic field $B$ [17]. The experimental gap behavior is in contradiction to the expected square-root dependence of the gap on magnetic field, which reveals problems with the straightforward consideration of electron-electron interactions in 2D electron systems.

In this paper, we perform measurements of the chemical potential jump across the fractional gap at filling factor $\nu=1 / 3$ and $\nu=2 / 5$ in the $2 \mathrm{D}$ electron system in $\mathrm{GaAs} / \mathrm{AlGaAs}$ single heterojunctions using a magnetocapacitance technique. The gap, $\Delta \mu$, increases with decreasing temperature and saturates becoming independent of temperature in the limit of low temperatures. In high magnetic fields, the limiting gap values, $\Delta \mu_{0}$, for $\nu=1 / 3$ and $\nu=2 / 5$ increase linearly with magnetic field so that the ratio of the slopes is equal, within the experimental uncertainty, to the inverse ratio of the fraction denominators. The temperature-independent difference between the $\mu$ values at $\nu=1 / 2$ and $\nu=1 / 4$ indicates that the fractional gap closes partially when the Fermi level lies outside the gap. Unlike the spin gap [18], the fractional gap decays due to temperature and disorder smearing. Using an empirical analysis of the data, we allow for the effect of sample inhomogeneities and extract the jump $\Delta \mu^{i d}(B)$ for an ideal $2 \mathrm{D}$ electron system. The results obtained suggest that in the highest accessible magnetic fields, the gap $\Delta \mu^{i d}$ is proportional to $q^{-1} B^{1 / 2}$. We reach the limit of very high magnetic fields where the expected square-root behavior prevails (in homogeneous samples), while discrepancies between experiment and theory may remain for lower $B$.

Measurements were made in an Oxford dilution refrigerator with a base temperature of $\approx 30 \mathrm{mK}$ on remotely doped GaAs/AlGaAs single heterojunctions (with a lowtemperature mobility $\approx 4 \times 10^{6} \mathrm{~cm}^{2} / \mathrm{Vs}$ at electron density $9 \times 10^{10} \mathrm{~cm}^{-2}$ ) having the quasi-Corbino geometry with area $2.2 \times 10^{5} \mu \mathrm{m}^{2}$. The depth of the $2 \mathrm{D}$ electron 

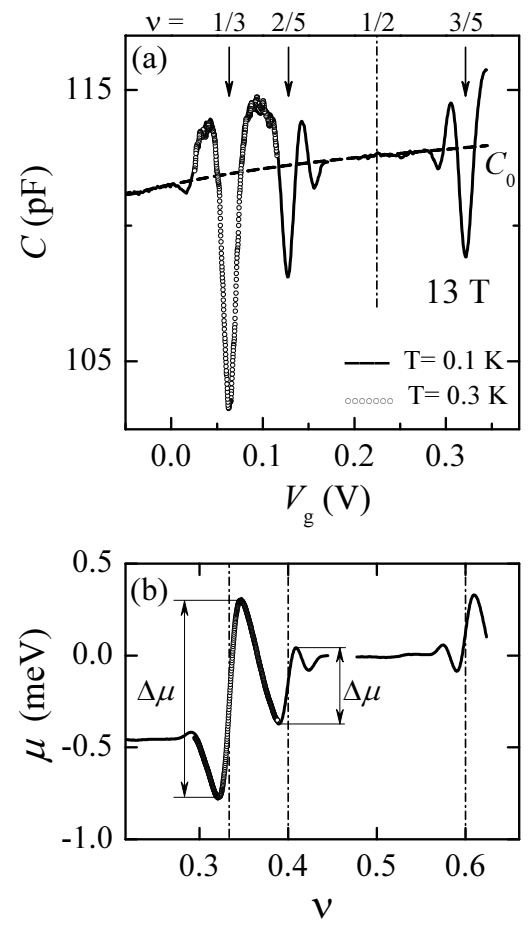

FIG. 1: (a) Magnetocapacitance as a function of gate voltage in sample 1 in $B=13 \mathrm{~T}$. Also shown by a dashed line is the geometric capacitance $C_{0}$. (b) The chemical potential as a function of $\nu$ obtained by integrating the magnetocapacitance; see text. The zero level corresponds to $\nu=1 / 2$.

layer was $210 \mathrm{~nm}$. A metallic gate was deposited onto the surface of the sample, which allowed variation of the electron density by applying a dc bias between the gate and the 2D electrons. The gate voltage was modulated with a small ac voltage of $2 \mathrm{mV}$ at frequencies in the range $0.05-11 \mathrm{~Hz}$, and both the imaginary and real components of the current were measured with high precision $\left(\sim 10^{-16} \mathrm{~A}\right)$ using a current-voltage converter and a lockin amplifier. Smallness of the real current component as well as proportionality of the imaginary current component to the excitation frequency ensure that we reach the low-frequency limit and the measured magnetocapacitance is not distorted by lateral transport effects. A dip in the magnetocapacitance in the quantum Hall state is directly related to a jump of the chemical potential across the corresponding gap in the spectrum of the $2 \mathrm{D}$ electron system [19]:

$$
\frac{1}{C}=\frac{1}{C_{0}}+\frac{1}{A e^{2} d n_{s} / d \mu}
$$

where $C_{0}$ is the geometric capacitance between the gate and the $2 \mathrm{D}$ electrons, $A$ is the sample area, and the derivative $d n_{s} / d \mu$ of the electron density over the chemical potential is the thermodynamic density of states.

A magnetocapacitance trace $C$ as a function of gate voltage $V_{g}$ is displayed in Fig. 1(a) for a magnetic field of 13 T. Narrow minima in $C$ accompanied at their edges by maxima are seen at filling factor $\nu \equiv n_{s} h c / e B=$
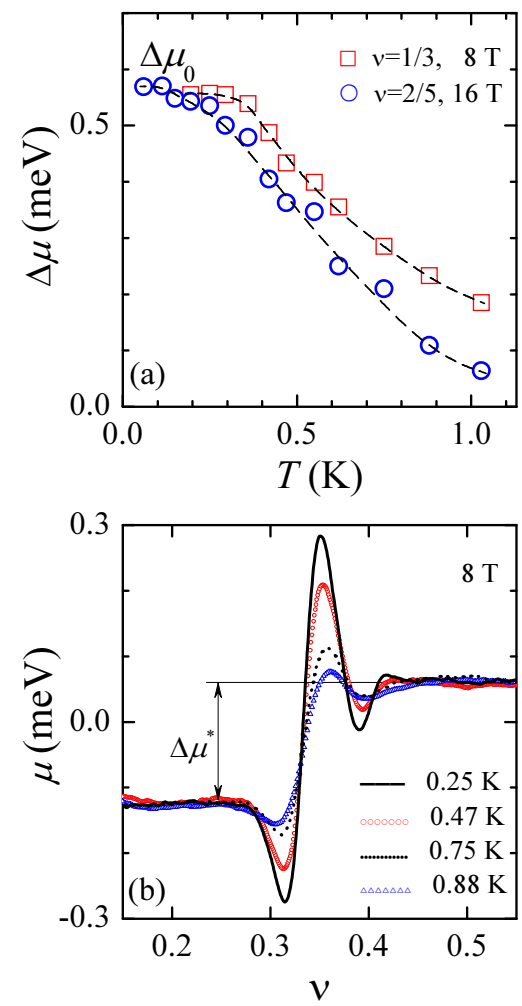

FIG. 2: (a) Temperature dependence of the fractional gap in sample 1 for $\nu=1 / 3$ at $B=8 \mathrm{~T}$ and for $\nu=2 / 5$ at $B=16 \mathrm{~T}$. The saturated low-temperature value $\Delta \mu_{0}$ is indicated. The dashed lines are guides to the eye. (b) The chemical potential as a function of $\nu$ at different temperatures. The zero level corresponds to $\nu=1 / 3$.

$1 / 3,2 / 5,3 / 5,2 / 7,3 / 7$, and $4 / 7$. Near the filling factor $\nu=1 / 2$, the capacitance $C$ in the range of magnetic fields studied reaches its high-field value determined by the geometric capacitance $C_{0}$ (dashed line). We have verified that the obtained $C_{0}$ corresponds to the value calculated using Eq. (1) from the zero-field capacitance and the density of states $m / \pi \hbar^{2}$ (where $m=0.067 m_{e}$ and $m_{e}$ is the free electron mass). The chemical potential jump $\Delta \mu$ for electrons at fractional filling factor can be determined by integrating the magnetocapacitance over the dip (for more details, see Ref. [17]):

$$
\Delta \mu=\frac{e}{C_{0}} \int_{\text {dip }}\left(C_{0}-C\right) d V_{g} .
$$

It is easy to determine the behavior of the chemical potential when the filling factor traverses the fractional gap, as shown in Fig. 1(b). The chemical potential jump corresponds to a cusp in the dependence of the groundstate energy, $E$, of the 2 D electron system on filling factor [2]: $\Delta \mu(\nu)=d E /\left.d n_{s}\right|_{\nu+0}-d E /\left.d n_{s}\right|_{\nu-0}$. The difference, $\Delta \mu^{*}$, between the $\mu$ values at $\nu=1 / 2$ and $\nu=1 / 4$ is smaller than $\Delta \mu(\nu=1 / 3)$, as determined by the maxima in the capacitance near $\nu=1 / 3$, see below. The values $\Delta \mu$ for $\nu=2 / 5$ and $\nu=3 / 5$ are equal (see also 


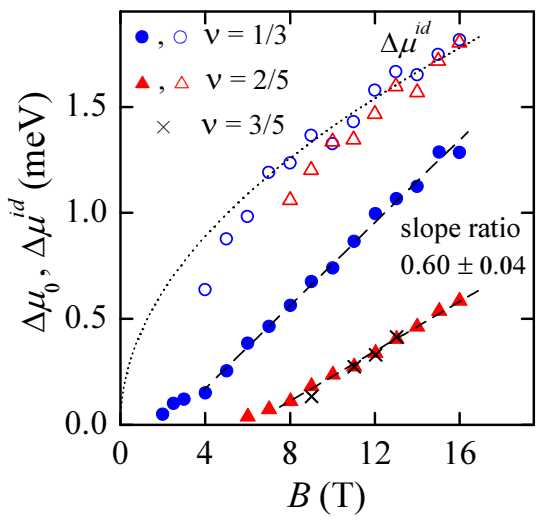

FIG. 3: The fractional gap $\Delta \mu_{0}$ (filled symbols and crosses) and jump $\Delta \mu^{i d}$ for an ideal $2 \mathrm{D}$ electron system (open symbols) versus $B$ in sample 1 . The dashed lines are linear fits to the high-field data. The slope ratio is equal, within the experimental uncertainty, to the inverse ratio of the fraction denominators. The value of $\Delta \mu^{i d}$ for $\nu=2 / 5$ is divided by the factor of 0.6. The dotted line is a square-root fit to the high-field data.

Fig. 3), which is already evident from Fig. 1(a): the difference $\delta C=C-C_{0}$ versus $\nu$ is nearly symmetric about $\nu=1 / 2$. The electron-hole symmetry in the spin-split Landau level is characteristic of the high-field data including the fractional features with $q=3,5$, and 7 .

In Fig. 2(a), we show the temperature dependence of the gap for $\nu=1 / 3$ and $\nu=2 / 5$. As the temperature is decreased, the value $\Delta \mu$ increases and in the limit of low temperatures, the gap saturates and becomes independent of temperature. It is the saturated low-temperature value $\Delta \mu_{0}$ that will be studied in the following as a function of the magnetic field.

In Fig. 2(b), we compare the temperature dependences of $\Delta \mu$ and $\Delta \mu^{*}$. While the jump $\Delta \mu$ decreases with temperature by more than a factor of two, the value $\Delta \mu^{*}$ does not change at all with increasing temperature up to $T \approx 0.9 \mathrm{~K}$. The qualitatively different behavior of both values with temperature allows us to conclude that the fractional gap closes partially (the value of $\Delta \mu_{0} / \Delta \mu^{*}$ is about three for $\nu=1 / 3$ ) when the Fermi level lies outside the gap. Since the gap $\Delta \mu$ decreases with both a deviation of the filling factor from fractional $\nu$ and temperature in a similar way (i.e., approximately linearly), its decay with temperature is likely to be caused by thermal smearing [20].

In Fig. 3, we show how the $\nu=1 / 3$ and $\nu=2 / 5$ gap $\Delta \mu_{0}$ changes with magnetic field. In high magnetic fields, the data are described by a linear increase of the gap value with $B$, which is consistent with the results of Ref. [17]. We find that the ratio of the slopes for $\nu=2 / 5$ and $\nu=1 / 3$ is the same, within the experimental uncertainty, as the inverse ratio of the fraction denominators, equal to $3 / 5$. This shows that the increase of the gap with magnetic field is determined by the denominator $q$.

In real samples, the jump of the chemical potential

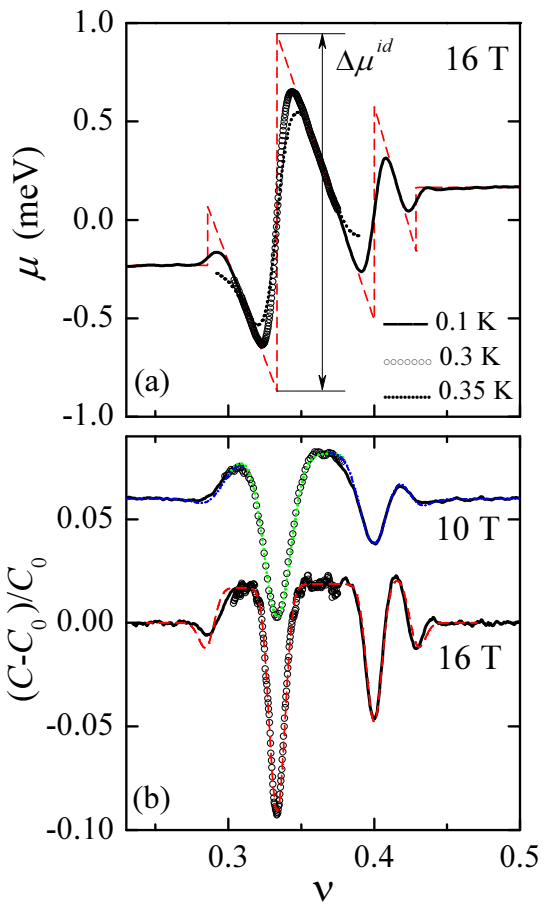

FIG. 4: (a) Dependence of the chemical potential on $\nu$ in the low-temperature limit in samples 1 (solid line and circles) and 2 (dotted line) in $B=16 \mathrm{~T}$. The dashed red line is a saw-tooth function obtained by the linear extrapolation to fractional $\nu$ of the intervals in which the quantity $\mu$ decreases with filling factor. The zero level corresponds to $\nu=1 / 3$. (b) Magnetocapacitance as a function of $\nu$ in the low-temperature limit in sample 1 (solid line and circles) at $B=16 \mathrm{~T}$ and $B=10 \mathrm{~T}$ (vertically shifted for clarity) and the fit based on convolution of the saw-tooth function with the Gaussian density distribution with $\sigma\left(n_{s}\right)=2 \times 10^{9} \mathrm{~cm}^{-2}$ (dashed red line) and the fit based on the experimental $\mu(\nu)$ in $B=16 \mathrm{~T}$ (dotted green and dash-dotted blue lines); see text.

$\Delta \mu_{0}$ is washed out due to inhomogeneities in the electron density distribution. Unlike the capacitance minima at integer $\nu$ [18], the fractional minima are surrounded by the relatively big maxima in $C$, which leads to a noticeable reduction of the disorder-broadened jumps of $\mu$ with respect to those in an ideal $2 \mathrm{D}$ electron system. An attempt to extract values of fractional gap corresponding to the clean limit was made in Ref. [11], based on the theoretical behavior of the chemical potential around fractional $\nu$ taking into account the electron-electron interactions. The results obtained do not confirm the expected square-root dependence of the gap on magnetic field and, therefore, one should treat electron-electron interactions in the fractional quantum Hall effect in a less straightforward way.

In Fig. 4(a), we compare the dependence $\mu(\nu)$ for the highest $B=16 \mathrm{~T}$ with that in another sample made of the same wafer but having a considerably higher level of inhomogeneities in the electron density distribution, as signaled by more broadened and less deep capacitance minima. Outside the $\nu=1 / 3$ gap, the decrease of the 
chemical potential with filling factor, which corresponds to the gap closing, can be described by a linear dependence, the same for both samples. Extrapolation of this linear behavior to $\nu=1 / 3$ yields an estimate of the jump $\Delta \mu^{i d}$ for an ideal 2D electron system.

The functional dependence of the gap $\Delta \mu^{i d}$ on magnetic field can be determined under the assumption that the shape of the oscillations $\mu^{i d}(\nu)$ does not depend explicitly on $B$ and their amplitude is a function of the magnetic field: behavior that is consistent with theoretical considerations discussed in detail in Ref. 11]. For the Gaussian density distribution with constant width $\sigma\left(n_{s}\right)$, the oscillations $\mu^{i d}(\nu)$ are progressively washed out with decreasing magnetic field. Since $\mu^{i d}(\nu)$ near the fractional $\nu$ is in fact unknown, we use the least-broadened experimental $\mu(\nu)$ in the highest $B=16 \mathrm{~T}$ for convolution in lower magnetic fields. By tuning the width $\sigma(\nu)=\sigma\left(n_{s}\right) h c / e B$ and varying the oscillation amplitude, we attain good fits to the measured $C(\nu)$ around $\nu=1 / 3$ and $\nu=2 / 5$ at $B<16 \mathrm{~T}$ (Fig. 4 (b)) and, thus, extract the behavior of $\Delta \mu^{i d}$ with $B$, as shown in Fig. 3. The procedure is applicable in high magnetic fields, whereas in low $B$ the quality of the fits is poor, making the determination of $\Delta \mu^{i d}(B)$ impossible. Note that the absolute value of $\Delta \mu^{i d}$ in Fig. 3 is an estimate obtained using linear extrapolations of the data (the dashed line in Fig. 4(a)); such saw-tooth oscillations $\mu^{i d}(\nu)$ with zero-width jumps at fractional $\nu$, convolved with the Gaussian density distribution with $\sigma\left(n_{s}\right)=2 \times 10^{9} \mathrm{~cm}^{-2}$ provide a high-quality fit to the experimental magnetocapacitance, shown in Fig. 目(b).

As seen from Fig. 3, the $\Delta \mu^{i d}(B)$ dependence approaches, ignoring the numerical factor, the theoretical square-root behavior of the fractional gap with $B$ in the high-field limit where the ratio of the jumps $\Delta \mu^{i d}$ for $\nu=2 / 5$ and $\nu=1 / 3$ is approximately equal to $3 / 5$. In other words, our results suggest that in the highest accessible magnetic fields, the gap $\Delta \mu^{i d}$ is proportional to $q^{-1} B^{1 / 2}$. Thus, we reach the limit of very high magnetic fields where the expected square-root behavior prevails (in homogeneous samples), while discrepancies between experiment and theory may remain for lower $B$.

In summary, we have studied the variation of the chemical potential in the 2D electron system in GaAs/AlGaAs single heterojunctions with changing filling factor around $\nu=1 / 3$ and $\nu=2 / 5$ and determined the fractional gap in the limit of low temperatures. In high magnetic fields, both gaps increase linearly with magnetic field with slopes proportional to the inverse fraction denominator. As inferred from the qualitatively different behavior with temperature of the jump $\Delta \mu$ and the difference of the $\mu$ values at $\nu=1 / 2$ and $\nu=1 / 4$, the fractional gap closes partially when the Fermi level lies outside the gap. Unlike the spin gap [18], the fractional gap decays due to temperature and disorder smearing. Using an empirical analysis of the data, we allow for the effect of sample inhomogeneities and extract the jump $\Delta \mu^{i d}(B)$ for an ideal 2D electron system. The results suggest that in the highest accessible magnetic fields, the fractional gap in the clean limit is inversely proportional to the denominator and follows the square-root behavior with magnetic field.

We gratefully acknowledge discussions with S. V. Kravchenko and M. P. Sarachik. We would like to thank J. P. Kotthaus for an opportunity to use the clean room facilities at LMU Munich and C. Roessler and C. Paulus for technical assistance. This work was supported by the RFBR, RAS, and the Programme "The State Support of Leading Scientific Schools". VSK acknowledges the A. von Humboldt foundation.
[1] D. C. Tsui, H. L. Stormer, and A. C. Gossard, Phys. Rev. Lett. 48, 1559 (1982).

[2] T. Chakraborty, Adv. Phys. 49, 959 (2000).

[3] N. d'Ambrumenil and R. Morf, Phys. Rev. B 40, 6108 (1989).

[4] C. Gros and A. H. MacDonald, Phys. Rev. B 42, 9514 (1990).

[5] B. I. Halperin, P. A. Lee, and N. Read, Phys. Rev. B 47, 7312 (1993).

[6] R. B. Laughlin, Phys. Rev. Lett. 50, 1395 (1983).

[7] J. K. Jain, Phys. Rev. Lett. 63, 199 (1989).

[8] B. I. Halperin, Phys. Rev. Lett. 52, 1583 (1984).

[9] G. S. Boebinger, A. M. Chang, H. L. Stormer, and D. C. Tsui, Phys. Rev. Lett. 55, 1606 (1985).

[10] A. F. Dethlefsen, E. Mariani, H. P. Tranitz, W. Wegscheider, and R. J. Haug, Phys. Rev. B 74, 165325 (2006).

[11] J. P. Eisenstein, L. N. Pfeiffer, and K. W. West, Phys. Rev. Lett. 68, 674 (1992); Phys. Rev. B 50, 1760 (1994).

[12] S. I. Dorozhkin, G. V. Kravchenko, R. J. Haug, K. v. Klitzing, and K. Ploog, JETP Lett. 58, 834 (1993).

[13] C. F. Hirjibehedin, A. Pinczuk, B. S. Dennis, L. N. Pfeif- fer, and K. W. West, Phys. Rev. Lett. 91, 186802 (2003).

[14] C. F. Hirjibehedin, I. Dujovne, A. Pinczuk, B. S. Dennis, L. N. Pfeiffer, and K. W. West, Phys. Rev. Lett. 95, 066803 (2005).

[15] R. R. Du, H. L. Stormer, D. C. Tsui, L. N. Pfeiffer, and K. W. West, Phys. Rev. Lett. 70, 2944 (1993).

[16] F. Schulze-Wischeler, E. Mariani, F. Hohls, and R. J. Haug, Phys. Rev. Lett. 92, 156401 (2004).

[17] V. S. Khrapai, A. A. Shashkin, M. G. Trokina, V. T. Dolgopolov, V. Pellegrini, F. Beltram, G. Biasiol, and L. Sorba, Phys. Rev. Lett. 99, 086802 (2007).

[18] V. T. Dolgopolov, A. A. Shashkin, A. V. Aristov, D. Schmerek, W. Hansen, J. P. Kotthaus, and M. Holland, Phys. Rev. Lett. 79, 729 (1997); V. S. Khrapai, A. A. Shashkin, E. L. Shangina, V. Pellegrini, F. Beltram, G. Biasiol, and L. Sorba, Phys. Rev. B 72, 035344 (2005).

[19] T. P. Smith, B. B. Goldberg, P. J. Stiles, and M. Heiblum, Phys. Rev. B 32, 2696 (1985); T. P. Smith III, W. I. Wang, and P. J. Stiles, Phys. Rev. B 34, 2995 (1986).

[20] M. O. Dorokhova and S. I. Dorozhkin, JETP 98, 1222 
(2004). 